\title{
On the linear increase of the flux tube thickness near the deconfinement transition.
}

\author{
A. Allais and M. Caselle \\ a Dipartimento di Fisica Teorica dell'Università di Torino and I.N.F.N., \\ via P.Giuria 1, I-10125 Torino, Italy \\ e-mail:_ (allais)(caselle)@to.infn.it
}

\begin{abstract}
We study the flux tube thickness of a generic Lattice Gauge Theory near the deconfining phase transition. It is well known that the effective string model predicts a logarithmic increase of the flux tube thickness as a function of the interquark distance for any confining LGT at zero temperature. It is perhaps less known that this same model predicts a linear increase in the vicinity of the deconfinement transition. We present a precise derivation of this result and compare it with a set of high precision simulations in the case of the $3 \mathrm{~d}$ gauge Ising model.
\end{abstract}




\section{Introduction}

The distinctive feature of the interquark potential in a confining gauge theory is that the colour flux is confined into a thin flux tube, joining the quark-antiquark pair. The quantum fluctuations of this flux tube can be well described by an effective string model. The most famous result of this model is the well known "Lüscher term" which was predicted more than 25 years ago $[1,2]$ and was recently observed in high precision montecarlo simulations of lattice gauge theories (LGTs) both in $(2+1)$ and in $(3+1)$ dimensions with gauge groups ranging from $Z_{2}$ to $S U(N)$ [3-15].

Another well known prediction of the effective string theory is the logarithmic increase of the width of the flux tube as a function of the interquark distance $R$. This behaviour was predicted many years ago by Lüscher, Münster and Weisz in [16] and subsequently observed in various different models, ranging again from $\mathrm{SU}(2)$ to $Z_{2}$ and to the pure gauge percolation model $[15,17-22]$. Together with the linear rising of the interquark potential it has always been considered as one of the distinctive features of the confining regime in LGTs.

A natural question is what happens of the flux tube width as the deconfinement temperature is approached from below. According to the above picture one would naively expect that the log behaviour should hold in the whole confining phase. However we shall show in this paper that this is not the case. More precisely, we shall show by means of high precision montecarlo simulations in the $(2+1)$ dimensional $Z_{2}$ gauge model, that the flux tube width also depends on the finite temperature of the theory and that near the deconfinement temperature (but still in the confining phase) the flux tube width increases linearly (and not logarithmically!) as a function of $R$.

As we shall see, this result is in perfect agreement with the effective string picture which indeed predicts a logarithmic increase at low temperature but shows a much more complex behaviour as the temperature increases and ultimately leads, as the deconfinement temperature is approached, to the linear behaviour observed in the simulations.

This paper is organised as follows. In sect. 2 we define the flux tube thickness and discuss its evaluation in the framework of the effective string model both at zero and at finite temperature. In sect. 3 we present our montecarlo simulation while in sect. 4 we discuss our results and compare them with the effective string predictions. Sect. 5 is devoted to a few concluding remarks. 


\section{Effective string prediction for the flux tube thick- ness}

\subsection{Definition of the flux tube thickness}

The lattice operator which is commonly used to evaluate the flux at zero temperature through a plaquette $p$ of the lattice is:

$$
\langle\phi(p ; W)\rangle=\frac{\left\langle W U_{p}\right\rangle}{\langle W\rangle}-\left\langle U_{p}\right\rangle
$$

where $W$ denotes a Wilson loop while $U_{p}$ denotes the operator associated with the plaquette $p$.

In a finite temperature setting we must substitute the Wilson loop with a pair of Polyakov loops. The lattice operator becomes in this case:

$$
\left\langle\phi\left(p ; P, P^{\prime}\right)\right\rangle=\frac{\left\langle P P^{\prime \dagger} U_{p}\right\rangle}{\left\langle P P^{\prime \dagger}\right\rangle}-\left\langle U_{p}\right\rangle
$$

where $P, P^{\prime}$ are two Polyakov loops separated by $R$ lattice spacings.

Within this setting the flux $\left\langle\phi\left(p ; P, P^{\prime}\right)\right\rangle$ depends on the spacelike coordinates of the plaquette, on its orientation, on the separation $R$ of the Polyakov loops and on the length $L$ of the lattice in the timelike direction. It does not depend on the timelike coordinate of the plaquette. Different possible orientations of the plaquette $p$ measure different components of the flux. In the following we shall neglect this dependence which plays no role if one is interested in the $R$ dependence of the flux tube width. Furthermore, since we are mainly interested in the tube width half way between the two quarks, we restrict the plaquette to lie on the symmetry (hyper)plane half way between the two Polyakov loops. Under these conditions we have:

$$
\left\langle\phi\left(p ; P, P^{\prime}\right)\right\rangle=\langle\phi(\vec{h} ; R, L)\rangle
$$

where $\vec{h}$ denotes the displacement of $p$ from the $P P^{\prime}$ plane. Along each of the directions spanned by $\vec{h}$, the flux density shows a gaussian like shape (see for instance Fig. 2 of [18]). The width of this gaussian is the quantity which is usually denoted as "flux tube thickness":

$$
w^{2}(R, L)=\frac{\sum_{\vec{h}} \vec{h}^{2}\langle\phi(\vec{h} ; R, L)\rangle}{\sum_{\vec{h}}\langle\phi(\vec{h} ; R, L)\rangle}
$$


This quantity only depends on the interquark distance $R$ and on the lattice size in the compactified timelike direction $L$, i.e. on the inverse temperature of the model. By tuning $L$ we can thus study the flux tube thickness near the deconfinement transition.

\subsection{Effective string model for the interquark potential}

The starting point of the effective string description of the interquark potential is to model the latter in terms of a string partition function:

$$
\left\langle P P^{\prime \dagger}\right\rangle=\int[\mathcal{D} h] e^{-S_{\mathrm{eff}}} \equiv Z(R, L)
$$

where $S_{\text {eff }}$ denotes the effective action for the world sheet spanned by the string. In (4), the functional integration is done over world sheet configurations which have fixed boundary conditions along the space-like direction, and periodic boundary conditions along the compactified, time-like direction (the Polyakov lines are the fixed boundary of the string world sheet).

The simplest and most natural string model is the Nambu-Goto one, which assumes the string action $S_{\text {eff }}$ to be proportional to the area spanned by the string world sheet:

$$
S_{\mathrm{eff}}=\sigma \int d \tau \int d \varsigma \sqrt{g}
$$

where $g$ is the determinant of the two-dimensional metric induced on the world-sheet by the embedding in $\mathbb{R}^{d}$ and $\sigma$ is the string tension, which appears as a parameter of the effective model.

Eq. (5) is invariant with respect to reparametrization and Weyl transformations. The standard choice to deal with these symmetries is to choose the so called "physical gauge" (see [23] for more details) in which $g$ becomes a function of the transverse displacements of the string world-sheet only. These displacements (which we shall denote in the following as $h^{i}(\varsigma, \tau)$ are required to satisfy the boundary conditions relevant to the problem - in the present case, periodic b.c. in the compactified direction and Dirichlet b.c. along the interquark axis direction:

$$
h^{i}(\tau+L, \varsigma)=h^{i}(\tau, \varsigma) ; \quad h^{i}(\tau,-R / 2)=h^{i}(\tau, R / 2)=0 .
$$

As it is well known, this gauge choice is anomalous: 1 : rotational symmetry is broken at the quantum level unless the model is defined in the critical

\footnotetext{
${ }^{1}$ Another way to understand this anomaly is to notice that this gauge fixing implicitly assumes that the world-sheet surface is a single-valued function of $(\tau, \varsigma)$, i.e. overhangs, cuts, or disconnected parts are excluded.
} 
dimension $d=26$. However, this anomaly is known to vanish at large distances [24], and this suggests to use the "physical gauge" for an IR, effective string description also for $d \neq 26$.

If we restrict our attention to the $d=2+1$ case a further simplification occurs since only one transverse degree of freedom $(h)$ survives and the crossinteractions terms among different transverse degrees of freedom disappear. In the physical gauge, (5) takes the form:

$$
S[h]=\sigma \int_{-L / 2}^{L / 2} d \tau \int_{-R / 2}^{R / 2} d \varsigma \sqrt{1+\left(\partial_{\tau} h\right)^{2}+\left(\partial_{\varsigma} h\right)^{2}} .
$$

Since we expect this model to be correct in the large $R$ limit the standard way to deal with the square root term in the action is to perform a large $R$ expansion in powers of the dimensionless quantity $(\sigma R L)^{-1}$.

$$
S[h] \sim \sigma L R+\frac{\sigma}{2} \int_{-L / 2}^{L / 2} d \tau \int_{-R / 2}^{R / 2} d \varsigma(\nabla h)^{2}+O\left((\sigma L R)^{-1}\right)
$$

The first term is the classical contribution, it simply gives the area term in the interquark potential and we shall neglect it in the following. The second term is a standard gaussian action while the higher order $O\left((\sigma R L)^{-1}\right)$ contributions encode string self-interactions.

If we neglect in the expansion the string self-interaction terms (the so called "gaussian approximation"), then using standard results of $2 \mathrm{~d}$ conformal field theory (CFT) the partition function of the effective string model can be evaluated exactly leading to the well known Lüscher term. With some more effort also higher order terms (and in particular the quartic selfinteraction term written above) can be evaluated [25]

The resulting predictions have been compared with Montecarlo simulations of Polyakov loop correlators for different gauge theories in the last few years, showing a very good agreement at large distances and an increasing disagreement as smaller distances and/or higher temperatures (i.e. smaller values of $L$ ) were approached (see for instance [8]) 


\subsection{The effective string width in the gaussian approximation}

The effective string approach allows to compute the flux tube width according to the following definition:

$$
w^{2}(x ; R, L)=\frac{\int_{\mathcal{C}}[\mathcal{D} h] h^{i}(t, x) h^{i}(t, x) e^{-S[h]}}{\int_{\mathcal{C}}[\mathcal{D} h] e^{-S[h]}}
$$

where the sum is intended (as in the previous section) over all the surfaces bordered by the to Polyakov loops. Since the b.c. are periodic in the timelike direction, there is no dependence on $t$, and on setting $x=0$, i.e. half way between the two Polyakov loops, we obtain the effective string prediction for the flux tube as defined in (3) .

The action $S[h]$ should be in principle the whole Nambu-Goto action (7), but, as anticipated, we shall truncate it to its gaussian approximation (8). In this way the width becomes a correlator in a $2 \mathrm{~d}$ free bosonic theory:

$$
w^{2}(x ; R, L)=\left\langle h^{i}(t, x) h^{i}(t, x)\right\rangle
$$

This correlator is singular and must be regularized. The most natural choice is a point splitting regularization:

$$
w^{2}(x ; R, L)=\left\langle h^{i}(t, x) h^{i}(t+\epsilon, x+\epsilon)\right\rangle
$$

In fact, in the original lattice description, the flux density is evaluated using a plaquette operator which has an intrinsic size of the order of the lattice spacing. This ultraviolet scale is translated in the effective string description in the $\epsilon$ parameter of the point splitting regularisation.

The correlator in $(2.3)$ can be evaluated exactly (see the appendix). One can then perform an expansion in $\epsilon$ of the result. As expected the first term diverges logarithmically while the remaining is finite and describes the dependence of the result on the modular parameter $L / R$ of the cylinder.

It is important at this point to distinguish the two regimes: low and high temperature.

- At low temperature, i.e. in the regime in which $L>>R$ the flux tube width in the gaussian approximation is given by:

$$
\begin{gathered}
\sigma w^{2}(z)=-\frac{1}{2 \pi} \log \frac{\pi|\epsilon|}{2 R}+\frac{1}{2 \pi} \log \left|\theta_{2}(\pi \operatorname{Re} z / R) / \theta_{1}^{\prime}(0)\right| \\
q=e^{-\pi L / 2 R}
\end{gathered}
$$


where (as discussed in the appendix) we use a complex coordinate $z$ to describe the cylinder bordered by the two Polyakov loops (with $\operatorname{Re} z$ representing the spacelike direction and $\operatorname{Im} z$ the timelike one) which are fixed in the positions $\operatorname{Re} z= \pm R / 2$. Setting $R_{c}=\pi|\epsilon| / 2$ we see that the dominant term is:

$$
\sigma w^{2}(z)=\frac{1}{2 \pi} \log \frac{R}{R_{c}}
$$

as we anticipated, while the next to leading correction in the $L>>R$ limit turns out to be:

$$
\frac{1}{2 \pi} \log \left|\cos \left(\frac{\pi \operatorname{Re} z}{R}\right)\right|
$$

which vanishes if we choose $z=0$.

- In the opposite regime $L<<R$ (i.e. high $\mathrm{T}$, but still in the confining phase) the flux tube width has a very different expression:

$$
\begin{gathered}
\sigma w^{2}(z)=-\frac{1}{2 \pi} \log \frac{\pi|\epsilon|}{L}+\frac{1}{2 \pi} \log \left|\theta_{4}(2 \pi i \operatorname{Re} z / L) / \theta_{1}^{\prime}(0)\right|+\frac{(\operatorname{Re} z)^{2}}{L R} \\
q=e^{-2 \pi R / L}
\end{gathered}
$$

This can be obtained by a modular transformation of the previous result or by direct calculation (see the appendix). In this case in the $R>>L$ limit the dominant term turns out to be proportional to $\log L$ instead of $\log R$ and the (linear) $R$ dependence only appears in the first subleading correction. In fact setting $z=0$ and using the relation:

$$
\theta_{1}^{\prime}(0)=\theta_{2}(0) \theta_{3}(0) \theta_{4}(0)
$$

we obtain:

$$
\sigma w^{2}(z)=-\frac{1}{2 \pi} \log \frac{\pi|\epsilon|}{L}-\frac{1}{2 \pi} \log \left|\theta_{2}(0) \theta_{3}(0)\right| .
$$

Expanding this expression in powers of $q=e^{-2 \pi R / L}$ and setting $L_{c}=$ $\pi|\epsilon|$ we find 2 :

$$
\sigma w^{2}(z)=\frac{1}{2 \pi} \log \frac{L}{L_{c}}+\frac{R}{4 L}-\frac{1}{\pi} e^{-2 \pi \frac{R}{L}}+\cdots
$$

\footnotetext{
${ }^{2}$ Notice a misprint in the analogous expression reported in [28] where the linear coefficient was erroneously quoted to be $1 / 6$ instead of $1 / 4$.
} 
This is the major result of this section and we shall devote the next sections to a check of this prediction with a set of high precision Montecarlo simulations.

\section{Montecarlo simulations}

Testing the logarithmic growth of the flux tube width with Montecarlo simulations is a very difficult task since it requires to study very large Wilson loops (or Polyakov loop correlators) and to control the statistical errors induced by the ratio of expectation values of (2)

Both these problems can be solved if one studies abelian LGTs for which a duality transformation can be implemented. In particular, for three dimensional LGTs, the dual model turns out to be a spin model. As discussed in [18] in this case one can study Polyakov loop correlators of arbitrary size by simply frustrating the links (in the dual lattice) orthogonal to the surface bordered by the Polyakov loops. The expectation value of the energy operator (which is dual to the plaquette of the original gauge theory) in this environment directly corresponds to the ratio of expectation values of (2) thus solving at the same time also the second problem mentioned above.

This strategy was adopted in [18] to study the thickness of flux tubes generated by Wilson loops in the $3 \mathrm{~d}$ gauge Ising model finding a perfect agreement with the predictions of the gaussian approximation discussed above. By choosing different couplings and different Wilson loop sizes the authors of [18] were able to test the log growth over a range of more than two orders of magnitude.

The present paper can be considered as a natural continuation of the above analysis in the case of the Polyakov loop geometry which, as mentioned above, allows to introduce into the analysis also the finite temperature scale and allows to study the crossover from a log to a linear growth of the flux tube thickness.

We report here for completeness a few details on the gauge Ising model, on the algorithm that we used and on the parameters that we used in our simulations.

\subsection{The gauge Ising model}

The $3 D \mathbb{Z}_{2}$ gauge model on a cubic lattice is defined through the partition function:

$$
Z_{\text {gauge }}(\beta)=\sum_{\left\{\sigma_{l}= \pm 1\right\}} \exp (-\beta S)
$$


where the action $S$ is a sum over all the plaquettes of the cubic lattice:

$$
S=-\sum_{\square} \sigma_{\square} \quad, \quad \sigma_{\square}=\sigma_{l_{1}} \sigma_{l_{2}} \sigma_{l_{3}} \sigma_{l_{4}} \quad .
$$

This model can be translated into the usual $3 D$ Ising model by the Kramers-Wannier duality transformation:

$$
\begin{aligned}
& Z_{\text {gauge }}(\beta) \propto Z_{\text {spin }}(\tilde{\beta}) \\
& \tilde{\beta}=-\frac{1}{2} \log [\tanh (\beta)]
\end{aligned}
$$

where $Z_{\text {spin }}$ is the partition function of the Ising model on the dual lattice:

$$
Z_{\text {spin }}(\tilde{\beta})=\sum_{s_{i}= \pm 1} \exp (-\tilde{\beta} H(s))
$$

with:

$$
H(s)=-\sum_{\langle i j\rangle} J_{\langle i j\rangle} s_{i} s_{j}
$$

where $i$ and $j$ denote nodes of the dual lattice and the sum is extended to the links $\langle i j\rangle$ connecting the nearest-neighbour sites. For the moment the couplings $J_{\langle i j\rangle}$ are all chosen equal to +1 .

Using the duality transformation it is possible to build up a one-to-one mapping of physical observables of the gauge system onto the corresponding spin quantities. For instance, the vacuum expectation value of Polyakov loops correlator can be expressed in terms of spin variables as follows. First, choose an arbitrary surface $\Sigma$ bounded by the two Polyakov loops; then "frustrate" the links of the dual lattice intersecting $\Sigma$, i.e. take $J_{\langle i j\rangle}=-1$ whenever $\langle i j\rangle \cap \Sigma \neq \emptyset$. Let us denote with $H^{\prime}(s)$ the Ising Hamiltonian with this choice of couplings: the new Ising partition function $Z_{\text {spin }}^{\prime}(\tilde{\beta})=$ $\sum_{s_{i}= \pm 1} \exp \left(-\tilde{\beta} H^{\prime}(s)\right)$ describes a vacuum modified by the two Polyakov loops, which we shall call the $\mathrm{P}$-vacuum. It is easy to see at this point that, thanks to duality we can write the expectation value of the Polyakov loops correlator as:

$$
\left\langle P P^{\prime \dagger}\right\rangle=\frac{Z_{\text {spin }}^{\prime}}{Z_{\text {spin }}}=\left\langle\prod_{\langle i j\rangle \cap \Sigma \neq \emptyset} \exp \left(-2 \tilde{\beta} s_{i} s_{j}\right)\right\rangle_{\text {spin }}
$$

where the product is over all the dual links intersecting $\Sigma$. 
Similarly it is easy to see that:

$$
\frac{<P P^{\prime \dagger} U_{p}>}{<P P^{\prime \dagger}>}=\left\langle\exp \left(-2 \tilde{\beta} s_{k} s_{l}\right)\right\rangle_{P}
$$

where $s_{k} s_{l}$ is the link dual to the plaquette $U_{p}(x)$ and $<\quad>_{P}$ denotes a mean value in the $\mathrm{P}$-vacuum.

In this way we can immediately obtain the flux density by simply looking at the mean value of the (dual of the) plaquette in the model in which all the links dual to $\Sigma$ are frustrated (see fig 1).

More precisely we have:

$$
\left\langle\phi\left(p, P, P^{\prime}\right)\right\rangle=\left\langle U_{p}\right\rangle_{P}-\left\langle U_{p}\right\rangle
$$

\subsection{Simulation setting}

We simulated the Ising model (both with and without frustrations) with a standard Swendsen-Wang algorithm. We chose to measure the flux with a plaquette parallel to the surface joining the two Polyakov loops (i.e. in the dual lattice, looking at the product of the spins joined by a link orthogonal to such surface, see Fig. 1). We directly evaluated from the simulations the flux tube thickness and used a jackknife procedure to estimate the statistical errors. We performed all the simulations on a lattice of size $80 \times 80 \times L$. We chose $\beta=0.75180$ for which the deconfinement transition is known to be located exactly at $L=8$ [26]. Another reason for this choice is that for this value of $\beta$ the string tension is known with very high precision (see [10]) $\sigma=$ $0.0105255(11)$. We extracted the flux tube width for values of the interquark distance $R$ ranging 3 from 5 to 50 and for $L \in\{9,10,11,12,14,16\}$, i.e. for values of the ratio $T / T_{c}$ ( $T_{c}$ being the critical temperature) ranging from $T / T_{c}=1 / 2$ to $T / T_{c}=8 / 9$.

\section{Results}

We report the results of our simulations in Tab. 1 and Tab. 2, The same data are plotted in Fig. 2

Looking at Fig. 2, it is easy to see that, in agreement with our effective string calculations, the data for $L>9$ show a very nice linear behaviour

\footnotetext{
${ }^{3}$ Due to the finite extent of the lattice size in the space directions $(L=80)$ we expect finite size corrections to become important for $R>50$. In order to check this expectation we simulated for one $L$ value: $L=10$ also a few values of $R$ larger than 50 .
} 


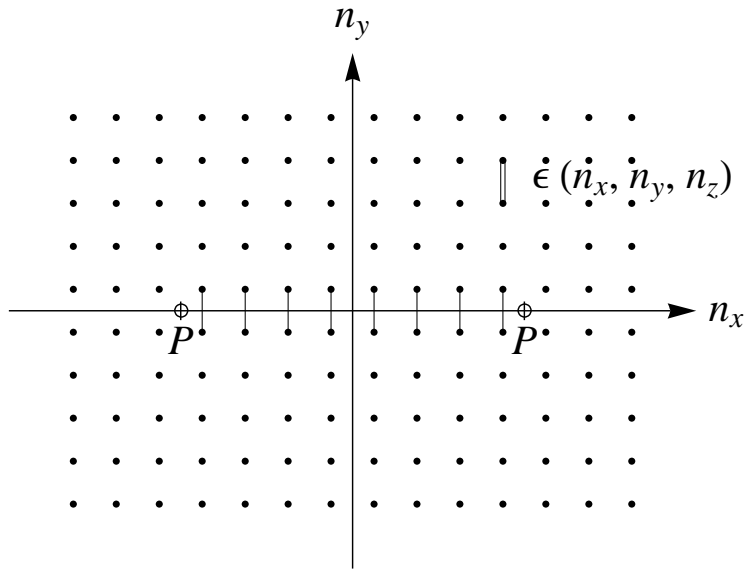

Figure 1: Schematic view of the simulation setting. The vertical bonds along the horizontal axis represent the frustrated links between the two Polyakov loops. The isolated bold face link represents the (dual of) plaquette operator.

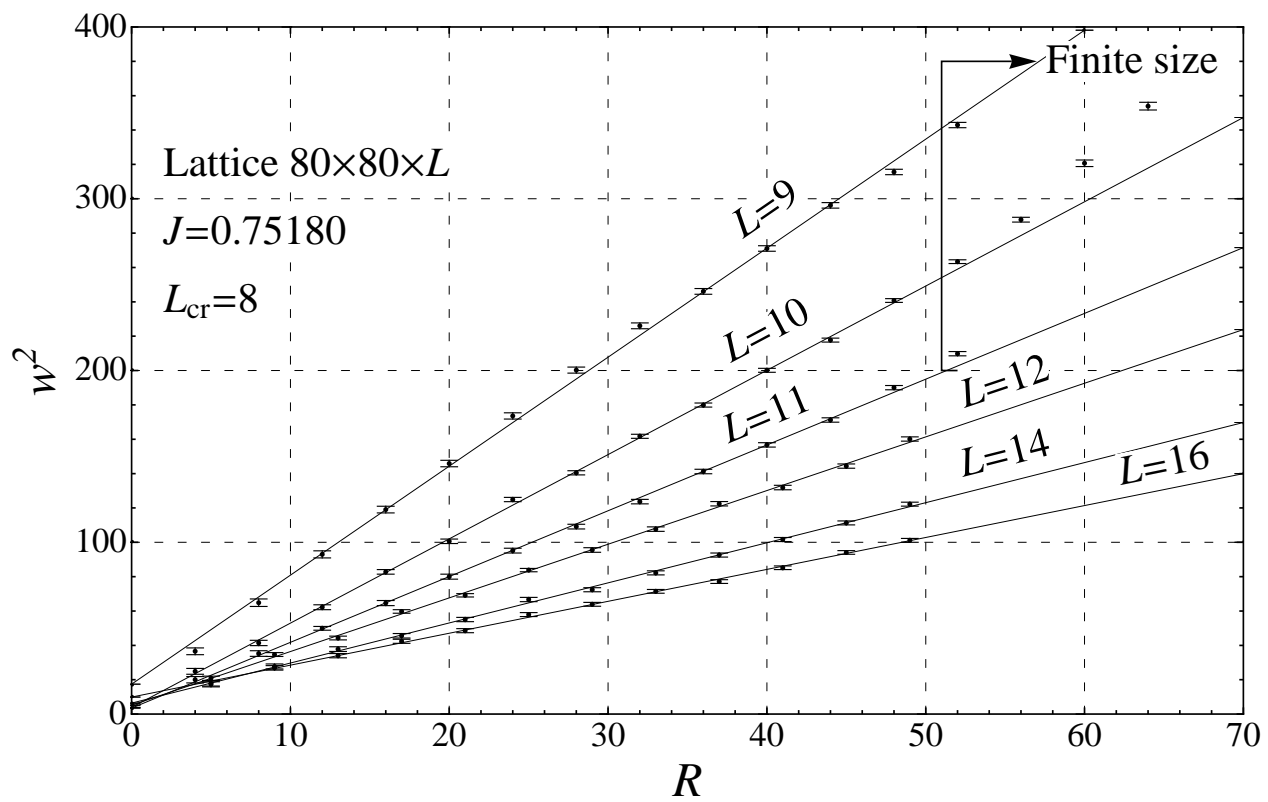

Figure 2: Flux tube thickness as a function of the interquark distance for various values of the inverse temperature $L$. 


\begin{tabular}{|c|c|c||c|c|c||c|c|c|}
\hline$L$ & $R$ & $w^{2}$ & $L$ & $R$ & $w^{2}$ & $L$ & $R$ & $w^{2}$ \\
\hline \hline 9 & 4 & $36.6(2.4)$ & 10 & 4 & $24.9(2.0)$ & 11 & 4 & $20.0(2.3)$ \\
9 & 8 & $64.8(2.6)$ & 10 & 8 & $41.4(1.8)$ & 11 & 8 & $35.2(2.0)$ \\
9 & 12 & $93.0(2.5)$ & 10 & 12 & $62.2(1.7)$ & 11 & 12 & $49.9(1.3)$ \\
9 & 16 & $119.0(2.4)$ & 10 & 16 & $82.8(1.6)$ & 11 & 16 & $64.6(1.8)$ \\
9 & 20 & $145.8(2.3)$ & 10 & 20 & $100.6(1.6)$ & 11 & 20 & $80.0(1.7)$ \\
9 & 24 & $173.6(2.3)$ & 10 & 24 & $124.9(1.6)$ & 11 & 24 & $95.1(1.7)$ \\
9 & 28 & $200.3(2.0)$ & 10 & 28 & $140.4(1.4)$ & 11 & 28 & $109.1(1.7)$ \\
9 & 32 & $226.0(2.0)$ & 10 & 32 & $161.7(1.4)$ & 11 & 32 & $123.7(1.6)$ \\
9 & 36 & $246.1(2.0)$ & 10 & 36 & $179.9(1.4)$ & 11 & 36 & $141.2(1.6)$ \\
9 & 40 & $271.0(1.9)$ & 10 & 40 & $200.1(1.3)$ & 11 & 40 & $156.7(1.6)$ \\
9 & 44 & $296.2(1.9)$ & 10 & 44 & $217.7(1.3)$ & 11 & 44 & $171.2(1.6)$ \\
9 & 48 & $315.6(1.9)$ & 10 & 48 & $240.8(1.3)$ & 11 & 48 & $190.0(1.4)$ \\
9 & 52 & $342.9(1.8)$ & 10 & 52 & $263.4(1.3)$ & 11 & 52 & $209.8(1.4)$ \\
& & & 10 & 56 & $287.8(1.7)$ & & & \\
& & & 10 & 60 & $320.7(2.3)$ & & & \\
& & & 10 & 64 & $353.9(2.6)$ & & & \\
\hline
\end{tabular}

Table 1: Results of the simulations: the square width $w^{2}$ as a function of $R$ and $L$

\begin{tabular}{|c|c|c||c|c|c||c|c|c|}
\hline$L$ & $R$ & $w^{2}$ & $L$ & $R$ & $w^{2}$ & $L$ & $R$ & $w^{2}$ \\
\hline \hline 12 & 5 & $20.9(1.6)$ & 14 & 5 & $17.9(1.9)$ & 16 & 5 & $17.3(1.8)$ \\
12 & 9 & $34.7(1.3)$ & 14 & 9 & $27.7(1.7)$ & 16 & 9 & $26.9(1.6)$ \\
12 & 13 & $44.3(1.3)$ & 14 & 13 & $37.8(1.7)$ & 16 & 13 & $34.0(1.6)$ \\
12 & 17 & $59.7(1.2)$ & 14 & 17 & $45.6(1.6)$ & 16 & 17 & $42.4(1.4)$ \\
12 & 21 & $69.1(1.2)$ & 14 & 21 & $55.0(1.6)$ & 16 & 21 & $48.6(1.4)$ \\
12 & 25 & $83.8(1.2)$ & 14 & 25 & $66.7(1.4)$ & 16 & 25 & $57.9(1.3)$ \\
12 & 29 & $95.5(1.6)$ & 14 & 29 & $72.4(1.4)$ & 16 & 29 & $63.9(1.3)$ \\
12 & 33 & $107.7(1.6)$ & 14 & 33 & $82.2(1.4)$ & 16 & 33 & $71.5(1.3)$ \\
12 & 37 & $122.4(1.6)$ & 14 & 37 & $92.6(1.4)$ & 16 & 37 & $77.1(1.3)$ \\
12 & 41 & $131.9(1.6)$ & 14 & 41 & $101.6(1.4)$ & 16 & 41 & $85.2(1.3)$ \\
12 & 45 & $144.4(1.6)$ & 14 & 45 & $111.3(1.4)$ & 16 & 45 & $94.1(1.3)$ \\
12 & 49 & $160.1(1.6)$ & 14 & 49 & $122.2(1.4)$ & 16 & 49 & $101.1(1.3)$ \\
\hline
\end{tabular}

Table 2: Same as Table 1 
as a function of $R$ in the range $10<R<50$. The data for $R>50$ show deviations due to finite size effects. We performed a linear fit of the data in the range $10<R<50$ with the law:

$$
w^{2}=k(L) R+c(L) ; \quad k(L)=\frac{k_{0}}{\sigma L}
$$

where, according to the effective string calculation, we should have $k_{0}=1 / 4$ The results are reported in Tab. 3 .

\begin{tabular}{|c|c|c|c|c|c|}
\hline$L$ & $k(L)$ & $c(L)$ & $k_{0}$ & $\sigma$ & $\chi_{r}^{2}$ \\
\hline \hline 9 & $6.19(10)$ & $23.0(3.2)$ & $0.587(16)$ & $4.48(7) \times 10^{-3}$ & 2.6 \\
10 & $4.90(4)$ & $4.1(1.5)$ & $0.516(5)$ & $5.10(5) \times 10^{-3}$ & 1.2 \\
11 & $3.85(4)$ & $2.6(1.2)$ & $0.446(4)$ & $5.90(6) \times 10^{-3}$ & 0.8 \\
12 & $3.14(4)$ & $4.4(1.2)$ & $0.397(5)$ & $6.63(8) \times 10^{-3}$ & 1.1 \\
14 & $2.33(3)$ & $6.3(1.2)$ & $0.344(5)$ & $7.64(11) \times 10^{-3}$ & 0.7 \\
16 & $1.84(3)$ & $10.6(9)$ & $0.309(4)$ & $8.50(12) \times 10^{-3}$ & 0.5 \\
\hline
\end{tabular}

Table 3: Results of the fit $w^{2}=k(L) R+c(L)$ for various values of $L$. In the fifth column we also report the values of the string tension $\sigma$ extracted from $k(L)$.

A few comments are in order on these fits:

1] We used the following criteria to fix the range of values of $R$ used in the fits. In order to fix the upper bound we performed a set of preliminary fits for the $L=10$ data keeping initially all the data and then iteratively discarding the largest ones looking for an acceptable $\chi_{r}^{2}$ (i.e. a reduced $\chi^{2}$ of order unity). In this way we identified as upper bound $R=50$. For the lower bound we used the same criterion adopted in previous works on effective string corrections which assumed the effective string to be a reliable description of the interquark potential for scales $R$ such that $\sigma R^{2} \geq 1$. In our case this means $R \geq 10$. Looking at (12) we see that with this choice the first subleading correction $\exp -2 \pi \frac{R}{L}$ turns out to be negligible within the statistical errors for all the values of $R$ and $L$ involved in the fits.

2] The linear fits show a reduced $\chi_{r}^{2}$ of order unity for all the values $L>9$ Also the data at $L=9$ show a linear like behaviour (see Fig. 22) which is however shadowed by rather large fluctuations. It is difficult to decide if this is the signature of a failure of the effective string picture or if the fluctuations are simply due to vicinity of the critical temperature. In any case we decided to neglect the $L=9$ data in the subsequent steps of our study. 
3] We can compare the values of $c(L)$ extracted from the fits with the effective string prediction:

$$
c(L)=\frac{1}{2 \pi \sigma} \log \frac{L}{L_{c}}
$$

We fitted the data for $L \geq 10$ with the law:

$$
c(L)=a \log L+b
$$

finding $a=17(4)$ and $b=-38(9)$ with a rather good value of $\chi_{r}^{2}=$ 1.6. This result turns out to be in remarkable agreement with the effective string prediction: $a=1 / 2 \pi \sigma=15.12 \ldots$ (obtained assuming $\sigma=0.0105241)$.

4] Looking at Tab. 3 we see that the values of $k(L)$ show a $L$ dependence different from the one predicted by the effective string. However the values that we obtain from the fits smoothly converge toward the predicted one as $L$ increase. This can be appreciated looking at the fourth column of Tab. 3 where we reported the values of $k_{0}$ extracted from the fits assuming $\sigma=0.0105241$ which should be compared with the effective string prediction $k_{0}=1 / 4$. For future utility we also reported in the fifth column of the table the values which we would predict for the string tension if we would fix $k_{0}=1 / 4$ in the fits. These values are plotted in Fig. 3,

\subsection{Discussion}

The above analysis shows that our data are in substantial agreement with the effective string predictions at large values of $R$ and $L$. At the same time however we see an increasing disagreement as $R$ and $L$ decrease. This is indeed an usual phenomenon when effective string predictions are compared with LGT data and points to the well known fact that the effective string only represents a large distance effective description of the interquark potential.

We see two main sources of disagreement. The first one, which we already discussed above, is the fact that the effective string correction $1 / 4$ in front of the $k(L)$ term is only reached asymptotically (see Fig. 3). The second one is that looking at the data in Tab. 1 and Tab. 2 we see that the linear behaviour as a function of $R$ extends also in the $R<10$ region. This definitely disagrees with the effective string prediction which in this region 


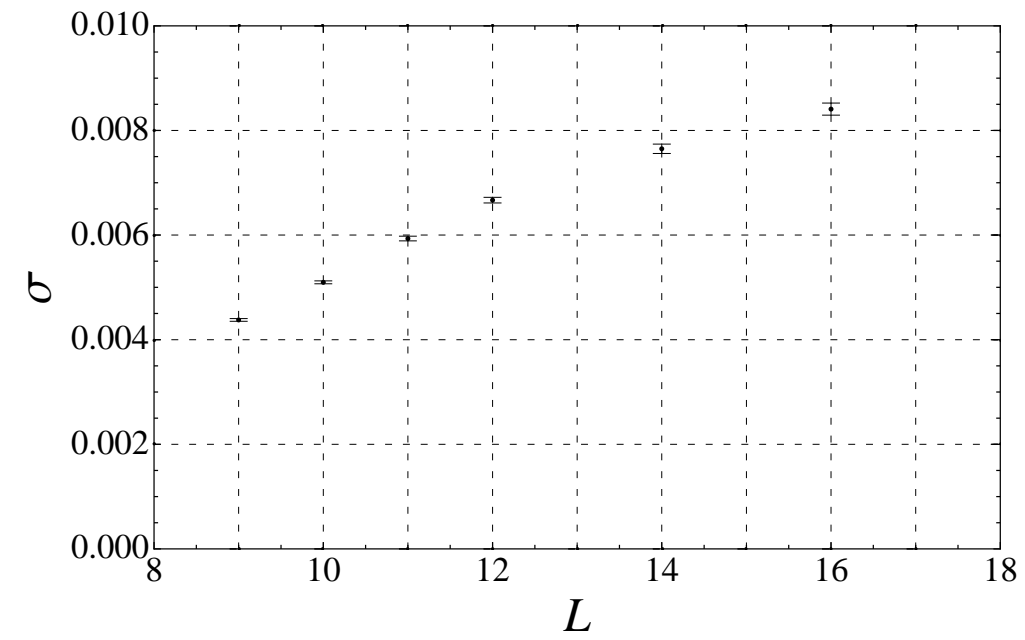

Figure 3: Plot of the string tension $\sigma$ extracted from $k(L)$ as a function of $L$.

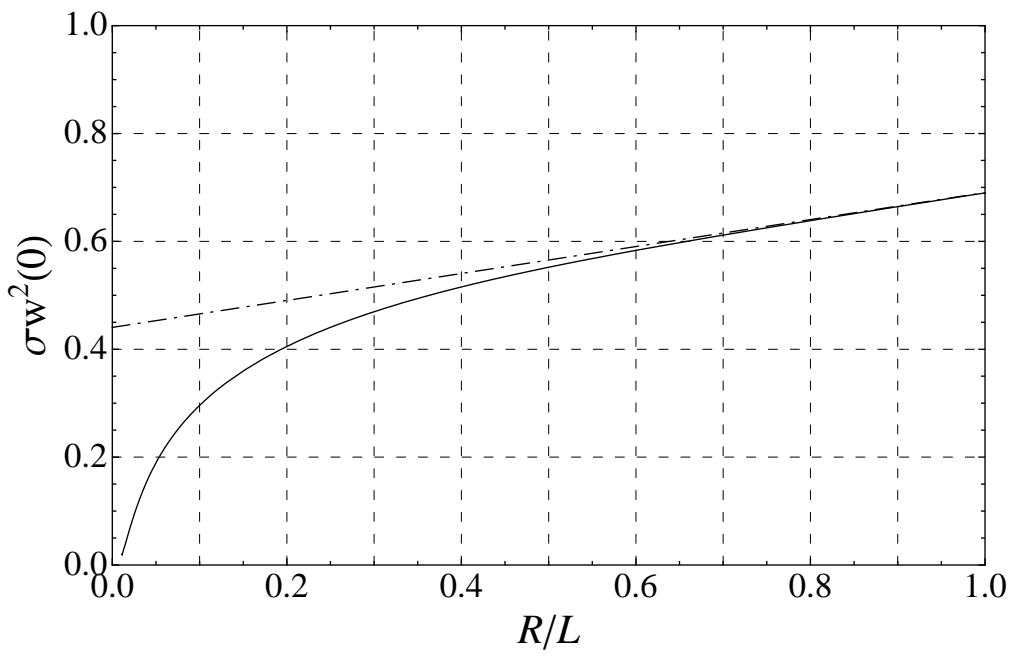

Figure 4: $R$ dependence of the flux tube width in the $R<L$ region. We also plot for comparison a straight line with angular coefficient $1 / 4$. 
(due to the higher terms in the theta functions) predicts instead a significant depletion of the flux tube width (see Fig. 4).

It is interesting to observe that both these behaviours instead agree with a naive Svetitsky-Yaffe [27] dimensional reduction picture (see. [28]). Indeed, according to this scheme, the correlation function of two Polyakov loops should behave as the spin-spin correlator of the $2 \mathrm{~d}$ Ising model and the combination of plaquette and Polyakov loops used to measure the flux tube thickness is mapped into the $\langle\epsilon \sigma \sigma\rangle$ correlator of the same Ising model. As discussed in [28] also in this framework one finds a linear increase of the (equivalent of the) flux tube width, which however in this dimensional reduction scheme holds for all values of $R$. Moreover the coefficient of this linear increase is proportional to $1 / m$ where $m$ is the mass of the equivalent $2 \mathrm{~d}$ Ising model and depends on $L$ in a way which strongly resembles the behaviour reported in Fig. 3 (see [28]).

It would be interesting to see if going beyond the gaussian approximation one could recover this behaviour also in the effective string framework. This would require extending the calculation reported in the appendix to higher orders of the Nambu-Goto action or alternatively to adapt to this problem the D-brane approach discussed in [29] to deal with the whole Nambu-Goto action. Work is in progress to test the feasibility of these approaches. 


\section{A Appendix A: Derivation of eq.(9,11)}

\section{A.1 The Green function for mixed boundary conditions}

The starting point of our analysis is the Green function for a free bosonic theory on a cylindric domain. This Green function is the solution of the Laplace equation in a $2 \mathrm{~d}$ rectangle with periodic boundary conditions in one direction and Dirichlet conditions in the other direction. This can be mapped to to an electrostatic problem and solved by the method of images. We report the result in this appendix for completeness and discuss in some detail a few of its properties which will later be relevant for the study of the flux tube thickness.

Let us map the cylinder onto a rectangle of the complex plane centered in the origin with sizes $\left[-L_{x} / 2, L_{x} / 2\right] \times\left[-L_{y} / 2, L_{y} / 2\right]$. Let us impose periodic boundary conditions along the imaginary axis, i.e. for $\operatorname{Im}(z)= \pm L_{y} / 2$ and Dirichlet b.c. along the real axis i.e. for $\operatorname{Re}(z)= \pm L_{x} / 2$.

Then the Green function can be written as:

$$
G\left(z ; z_{0}\right)=-\frac{1}{2 \pi} \log \left|f\left(z, z_{0}\right)\right|
$$

with:

$$
f\left(z ; z_{0}\right)=\frac{\theta_{1}\left[\pi\left(z-z_{0}\right) / 2 L_{x}\right]}{\theta_{2}\left[\pi\left(z+\bar{z}_{0}\right) / 2 L_{x}\right]}
$$

where the Jacobi theta functions $\theta_{1}$ and $\theta_{2}$ are defined as:

$$
\begin{gathered}
\theta_{1}(z)=2 q^{\frac{1}{4}} \sum_{n=0}^{\infty}(-1)^{n} q^{n(n+1)} \sin (2 n+1) z \\
\theta_{2}(z)=2 q^{\frac{1}{4}} \sum_{n=0}^{\infty} q^{n(n+1)} \cos (2 n+1) z
\end{gathered}
$$

with:

$$
q=e^{i \pi \tau} ; \quad \tau=i L_{y} / 2 L_{x}
$$

In fact, with this definition, $\log f$ is analytic everywhere in the rectangle except in $z=z_{0}$ where $f(z)=0$ and the Green function diverges logarithmically. For all the remaining values since $\log |f|=\operatorname{Re} \log f$ (i.e. it is the real part of an analytic function) it satisfies the Laplace equation:

$$
\Delta G\left(z, z_{0}\right)=0
$$


As for the boundary conditions, by using the transformation properties of the theta functions:

$$
\theta_{1}(z+\pi \tau)=-q^{-1} e^{-2 i z} \theta_{1}(z) ; \quad \theta_{2}(z+\pi \tau)=q^{-1} e^{-2 i z} \theta_{2}(z) ;
$$

one can immediately see that:

$$
G\left(z+i L_{y}, z_{0}\right)=G\left(z, z_{0}\right)
$$

i.e. that $G$ is periodic along the imaginary axis with period $i L_{y}$

In order to check that eq A.1A.2 satisfy the Dirichlet conditions along the $x$ axis, let us set $z=L_{x} / 2+i y$. With this choice we have:

$$
G\left(z ; z_{0}\right)=-\frac{1}{2 \pi} \log \left|\frac{\theta_{1}\left[\pi\left(i y-z_{0}\right) / 2 L_{x}+\pi / 4\right]}{\theta_{2}\left[\pi\left(i y+\bar{z}_{0}\right) / 2 L_{x}+\pi / 4\right]}\right|
$$

from which, using the identity $\theta_{2}(z)=\theta_{1}(\pi / 2-z)$ we immediately obtain:

$$
G\left(z ; z_{0}\right)=-\frac{1}{2 \pi} \log \left|\frac{\theta_{1}\left[\pi\left(i y-z_{0}\right) / 2 L_{x}+\pi / 4\right]}{\theta_{1}\left[\pi\left(-i y-\bar{z}_{0}\right) / 2 L_{x}+\pi / 4\right]}\right|
$$

Since the theta functions are real along the real axis, the denominator in the above equation is the complex conjugate of the numerator and the argument of the logarithm is always unity for any value of $y$. In a similar way one can show that the Dirichlet b.c. hold also for $\operatorname{Re} z=-L_{x} / 2$. The expansion of eq.s A.1A.2 converges very quickly when $L_{y}>>L_{x}$. In this limit the exponentially decreasing terms in $G\left(z, z_{0}\right)$ can be neglected and only the dominant terms in the theta functions give a contribution. However in the opposite limit $L_{x}>>L_{y}$ such expression is almost useless. In this regime one should better perform a modular transformation $\tau \rightarrow-1 / \tau$ of the above result. In this way one obtains:

$$
\begin{gathered}
G\left(z ; z_{0}\right)=-\frac{1}{2 \pi} \log \left|\frac{\theta_{1}\left[i \pi\left(z-z_{0}\right) / L_{y}\right]}{\theta_{4}\left[i \pi\left(z+\bar{z}_{0}\right) / L_{y}\right]}\right|+\frac{\operatorname{Re} z \operatorname{Re} z_{0}}{L_{x} L_{y}} \\
q=e^{-2 \pi L_{x} / L_{y}} ; \quad \tau=2 i L_{x} / L_{y}
\end{gathered}
$$

This expression is equivalent to the above one, but converges well in the $L_{x}>>L_{y}$ limit. 


\section{A.2 The flux tube width}

The behaviour of the flux tube width can be extracted from eq. (2.3) performing an expansion in $\epsilon$. Keeping into account the prefactor $\sigma$ in the gaussian effective action eq. (8) we see that eq. (2.3) can be rewritten as:

$$
\sigma w^{2}(x, y)=G(z, z+\epsilon)
$$

In the limit $\epsilon \rightarrow 0$, using eq.s A.1A.2 and setting $L_{x}=R, L_{y}=L$ we obtain:

$$
\begin{gathered}
\sigma w^{2}(z)=-\frac{1}{2 \pi} \log \frac{\pi|\epsilon|}{2 R}+\frac{1}{2 \pi} \log \left|\theta_{2}(\pi \operatorname{Re} z / R) / \theta_{1}^{\prime}\right| \\
q=e^{-\pi L / 2 R}
\end{gathered}
$$

This expression converges well when $L>>R$.

In the opposite regime $R>>L$ we must use eq. (A.3) which leads to:

$$
\begin{gathered}
\sigma w^{2}(z)=-\frac{1}{2 \pi} \log \frac{\pi|\epsilon|}{L}+\frac{1}{2 \pi} \log \left|\theta_{4}(2 \pi i \operatorname{Re} z / L) / \theta_{1}^{\prime}\right|+\frac{(\operatorname{Re} z)^{2}}{L R} \\
q=e^{-\pi 2 R / L}
\end{gathered}
$$

Acknowledgements. The authors would like to thank P.Grinza, F. Gliozzi, M. Billó and L. Ferro for useful discussions.

\section{References}

[1] M. Lüscher, K. Symanzik, and P. Weisz, Anomalies of the free loop wave equation in the WKB approximation, Nucl. Phys. B173 (1980) 365.

[2] M. Lüscher, Symmetry Breaking Aspects of the Roughening Transition in Gauge Theories, Nucl. Phys. B180 (1981) 317.

[3] P. de Forcrand, G. Schierholz, H. Schneider and M. Teper, The String and Its Tension in SU(3) Lattice Gauge Theory: Towards Definitive Results, Phys. Lett. B160 (1985) 137. 
[4] G. S. Bali and K. Schilling, Static quark-antiquark potential: Scaling behavior and finite size effects in SU(3) lattice gauge theory, Phys. Rev. D46 (1992) 2636.

[5] M. Lüscher and P. Weisz, Quark confinement and the bosonic string, JHEP 07 (2002) 049 [hep-lat/0207003.

[6] K. J. Juge, J. Kuti and C. Morningstar, Fine structure of the $Q C D$ string spectrum, Phys. Rev. Lett. 90 (2003) 161601 [hep-lat/0207004.

[7] P. Majumdar, The string spectrum from large Wilson loops, Nucl. Phys. B664 (2003) 213 [ hep-lat/0211038.

[8] M. Caselle, M. Hasenbusch and M. Panero, String effects in the $3 d$ gauge Ising model, JHEP 01 (2003) 057 [ hep-lat/0211012.

[9] M. Caselle, M. Pepe and A. Rago, Static quark potential and effective string corrections in the $(2+1) d S U(2)$ Yang-Mills theory, JHEP 10 (2004) 005 [ hep-lat/0406008.

[10] M. Caselle, M. Hasenbusch and M. Panero, The interface free energy: comparison of accurate Monte Carlo results for the $3 D$ Ising model with effective interface models, JHEP 09 (2007) 117 [ 0707.0055$].$

[11] M. Panero, A numerical study of confinement in compact QED, JHEP 05 (2005) 066 [hep-lat/0503024.

[12] B. Lucini and M. Teper, Confining strings in $S U(N)$ gauge theories, Phys. Rev. D64 (2001) 105019 [ hep-lat/0107007].

[13] F. Gliozzi, S. Lottini, M. Panero and A. Rago, Random percolation as a gauge theory, Nucl. Phys. B719 (2005) 255 [ cond-mat/0502339.

[14] A. Athenodorou, B. Bringoltz and M. Teper, The closed string spectrum of $S U(N)$ gauge theories in $2+1$ dimensions, Phys. Lett. B656 (2007) 132 [0709.0693].

[15] G. S. Bali, K. Schilling and C. Schlichter, Observing long color flux tubes in SU(2) lattice gauge theory, Phys. Rev. D51 (1995) 5165 [ hep-lat/9409005.

[16] M. Luscher, G. Munster and P. Weisz, How Thick Are Chromoelectric Flux Tubes?, Nucl. Phys. B 180 (1981) 1. 
[17] P. Pennanen, A. M. Green and C. Michael, Flux-tube structure and $\beta$ functions in SU(2), Phys. Rev. D56 (1997) 3903 [hep-lat/9705033.

[18] M. Caselle, F. Gliozzi, U. Magnea and S. Vinti, Width of long colour flux tubes in Lattice Gauge systems, Nucl. Phys. B460 (1996) 397 [ hep-lat/9510019.

[19] M. Zach, M. Faber and P. Skala, "Investigating confinement in dually transformed U(1) lattice gauge theory", Phys. Rev. D57 (1998) 123 [ hep-lat/9705019.

[20] Y. Koma, M. Koma and P. Majumdar, Static potential, force, and fluxtube profile in $4 D$ compact $U(1)$ lattice gauge theory with the multi-level algorithm, Nucl. Phys. B692 (2004) 209 [ hep-lat/0311016].

[21] M. Pfeuffer, G. S. Bali and M. Panero, "Fluctuations of the baryonic flux-tube junction from effective string theory," arXiv:0810.1649 [hepth].

[22] P. Giudice, F. Gliozzi and S. Lottini, "Quantum broadening of k-strings in gauge theories", JHEP 01 (2007) 084 [ hep-th/0612131.

[23] O. Alvarez, "The Static Potential In String Models," Phys. Rev. D 24, 440 (1981).

[24] P. Olesen, "Strings And QCD," Phys. Lett. B 160 (1985) 144.

[25] K. Dietz and T. Filk, "On The Renormalization Of String Functionals," Phys. Rev. D 27 (1983) 2944.

[26] M. Caselle and M. Hasenbusch, "Deconfinement transition and dimensional cross-over in the 3D gauge Ising model," Nucl. Phys. B 470 (1996) 435 arXiv:hep-lat/9511015].

[27] B. Svetitsky and L. G. Yaffe, "Critical Behavior At Finite Temperature Confinement Transitions," Nucl. Phys. B 210 (1982) 423.

[28] M. Caselle, P. Grinza and N. Magnoli, "Study of the flux tube thickness in 3d LGT's by means of 2d spin models," J. Stat. Mech. 0611 (2006) P003 arXiv:hep-th/0607014.

[29] M. Billo and M. Caselle, "Polyakov loop correlators from D0brane interactions in bosonic string theory," JHEP 0507 (2005) 038 arXiv:hep-th/0505201]. 